\title{
Declining Burden of Plasmodium vivax in a Population in Northwestern Thailand from 1995 to 2016 before Comprehensive Primaquine Prescription for Radical Cure
}

\author{
Cindy S. Chu, ${ }^{1,2 *} \dagger$ Verena I. Carrara, ${ }^{3} \dagger$ Daniel M. Parker, ${ }^{4}$ Stéphane Proux, ${ }_{1}^{1}$ Prakaykaew Charunwatthana, ${ }^{5,6}$ \\ Rose McGready, ${ }^{1,2}$ and François Nosten ${ }^{1,2}$ \\ ${ }^{1}$ Shoklo Malaria Research Unit, Mahidol-Oxford Tropical Medicine Research Unit, Faculty of Tropical Medicine, Mahidol University, Mae Sot, \\ Thailand; ${ }^{2}$ Centre for Tropical Medicine, Nuffield Department of Medicine, University of Oxford, Oxford, United Kingdom; ${ }^{3}$ Department of Medicine, \\ Swiss Tropical and Public Health Institute, Basel, Switzerland; ${ }^{4}$ Department of Population Health and Disease Prevention, University of California, \\ Irvine, California; ${ }^{5}$ Mahidol-Oxford Tropical Medicine Research Unit, Faculty of Tropical Medicine, Mahidol University, Mae Sot, Thailand; \\ ${ }^{6}$ Department of Clinical Tropical Medicine, Faculty of Tropical Medicine, Mahidol University, Bangkok, Thailand
}

\begin{abstract}
All Plasmodium cases have declined over the last decade in northwestern Thailand along the Myanmar border. During this time, Plasmodium vivax has replaced Plasmodium falciparum as the dominant species. The decline in $P$. falciparum has been shadowed by a coincidental but delayed decline in $P$. vivax cases. This may be due to early detection and artemisinin-based therapy, species-specific diagnostics, and bed net usage all of which reduce malaria transmission but not $P$. vivax relapse. In the absence of widespread primaquine use for radical cure against $P$. vivax hypnozoites, the decline in $P$. vivax may be explained by decreased hypnozoite activation of $P$. vivax relapses triggered by $P$. falciparum. The observed trends in this region suggest a beneficial effect of decreased $P$. falciparum transmission on $P$. vivax incidence, but elimination of $P$. vivax in a timely manner likely requires radical cure.
\end{abstract}

\section{INTRODUCTION}

Malaria has decreased in the Greater Mekong Subregion (GMS) over the last decade. The WHO estimates that from 2012 to 2015 , malaria cases declined by $54 \%$ and malariarelated mortality by $84 \%$ in this region. All GMS nations have drafted plans for malaria elimination by $2030 .{ }^{1}$ Because of the emergence of artemisinin-resistant parasites Plasmodium falciparum rather than Plasmodium vivax has been the focus of elimination. Plasmodium vivax remains a major contributor to morbidity largely because of relapses (as high as $80 \%$ in northwest Thailand). ${ }^{2}$ Treating relapses (radical cure) requires completing a prolonged primaquine course (7-14 days), leading to decreased adherence. Daily primaquine doses $(0.25-0.5 \mathrm{mg} /$ $\mathrm{kg} /$ day) needed for radical cure are contraindicated in persons with glucose-6-phosphate dehydrogenase (G6PD) deficiency because of hemolytic risk. ${ }^{3}$ When G6PD testing is not available, primaquine prescription is less likely. A weekly (for 8 weeks) regimen is recommended for G6PD-deficient individuals, which also limits adherence. Currently, primaquine is contraindicated during pregnancy and lactation. ${ }^{3}$ Consequently, the drug is often not prescribed, relapses are not prevented, and clinical infections recur after schizonticidal treatment.

Plasmodium falciparum malaria has decreased along the Thailand-Myanmar border over the last decade. ${ }^{4}$ Without widespread radical cure, a different pattern might be expected for $P$. vivax. Here, we report longitudinal trends in passively detected $P$. vivax infections in medical clinics along the Thailand-Myanmar border for a period spanning 22 years.

\section{METHODS AND RESULTS}

Shoklo Malaria Research Unit (SMRU) operates clinics located in Thailand along the Moei River, which forms the border

\footnotetext{
*Address correspondence to Cindy S. Chu, Shoklo Malaria Research Unit, Mahidol-Oxford Tropical Medicine Research Unit, Faculty of Tropical Medicine, Mahidol University, 68/30 Ban Toong Rd., \#46, Mae Sot 63110, Thailand. E-mail: cindy@tropmedres.ac

†These authors contributed equally to this work.
}

with Myanmar. The unit has field-based medical clinics, situated $30-120 \mathrm{~km}$ outside Mae Sot, Thailand, serving refugee and migrant (mostly unregistered) border populations. The refugee clinic operated from 1986 to 2016. The first migrant clinic was opened in 1998, second in 1999, and two additional clinics were opened in 2004-2005.

Malaria transmission in this area is low, unstable, and seasonal. ${ }^{5}$ The dominant species are $P$. falciparum and $P$. vivax, and Plasmodium malariae and Plasmodium ovale are uncommon. In patients with a fever history, a malaria smear was performed routinely in the refugee clinic, whereas in the migrant clinics malaria rapid diagnostic tests (RDTs) were also performed. Malaria smears in the migrant clinics were performed systematically in children aged $<5$ years and during a one-week period each month (called "smear week"). ${ }^{6}$ Before March 2010, the RDTs that were used diagnosed only $P$. falciparum so $P$. vivax was a clinical diagnosis (i.e., fever and RDT negative) during the non "smear weeks". When newer RDTs were used, all $P$. vivax diagnoses were confirmatory (RDTs or malaria smear). Malaria microscopy was performed by trained laboratory technicians. Standardized quality control for malaria smear and RDT reading was conducted routinely.

At SMRU, mefloquine-artesunate was the first-line treatment for $P$. falciparum and mixed $P$. falciparum infections from 1994 until June 2012 when it was changed to dihydroartemisinin-piperaquine plus gametocytocidal singledose primaquine. Chloroquine has remained efficacious against $P$. vivax. After 2010, routine radical cure for $P$. vivax was implemented, but only under direct supervision, resulting in $<10 \%$ of patients with $P$. vivax treated with primaquine. Until 2017, there were no targeted radical curative interventions in the population. However, from 2010 to 2014, a series of $P$. vivax studies were performed and primaquine was prescribed to $\sim 1,200$ participants. A P. falciparum elimination campaign in community-based malaria clinics using standard artemisinin-based therapy (ACTs) and single-dose primaquine began in 2014 in Kayin State, Myanmar, covering areas opposing Tak Province. In these community-based malaria 


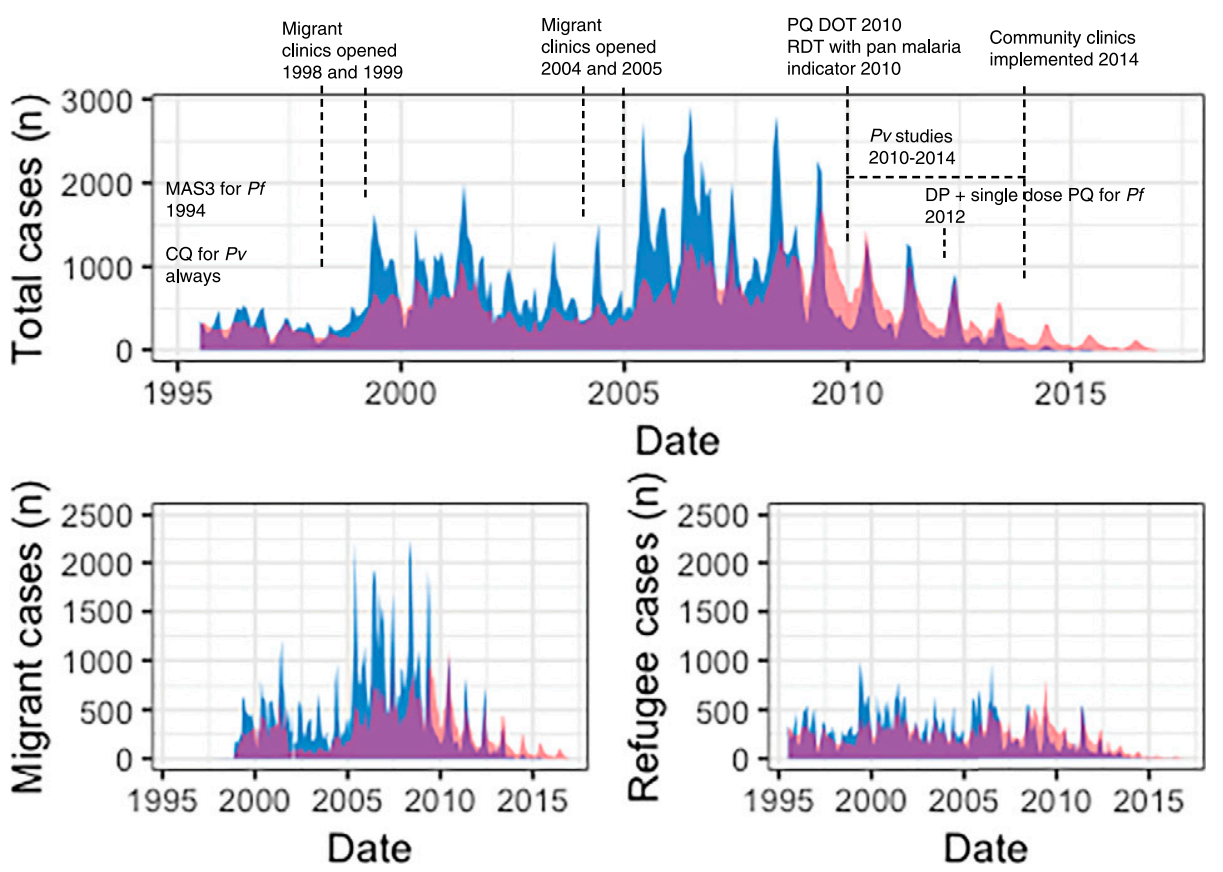

FIGURE 1. Number of cases of Plasmodium falciparum and Plasmodium vivax from 1995 to 2016 in the refugee and migrant clinics. Plasmodium falciparum is indicated in blue and Plasmodium vivax in red. $\mathrm{CQ}=$ chloroquine; $\mathrm{DP}=$ dihydroartemisinin piperaquine; MAS $3=\mathrm{mefloquine} 25 \mathrm{mg} / \mathrm{kg}$ total dose plus artesunate $4 \mathrm{mg} / \mathrm{kg} /$ day for 3 days; PQ DOT = primaquine directly observed treatment (in patients who agreed to follow up); RDT = rapid diagnostic test.

clinics, some overlapping the SMRU catchment area, radical cure for $P$. vivax was not prescribed. ${ }^{7,8}$

For this analysis, aggregated anonymized data from monthly reports on nonpregnant patients with $P$. falciparum and $P$. vivax malaria diagnosed in the SMRU medical clinics from July 1995 to December 2016 in refugees and November 1998 to December 2016 in migrants (before universal primaquine administration) were included. Mixed infections were attributed to both $P$. falciparum and $P$. vivax cases. In years with clinical $P$. vivax diagnosis, the annual caseload was adjusted by the proportion of $P$. vivax cases detected during the "smear weeks" of the same year. Descriptive statistics were used for $P$. vivax and $P$. falciparum cases by year, age (grouped every 5 years until 39 years, then all $\geq 40$ years), gender, and status (migrant or refugee). Poisson regressions were used to test for differences in trends in cases over time by agegroup, gender, and status. Then the data were stratified by gender with an interaction term between year and age-group to detect changing patterns in the distribution of cases across the lifespan, over time.

Overall, the number of malaria cases decreased (Figure 1). Increases in 1999 and 2005 coincided with opening new migrant clinics and peaked in 2006. By the end of 2010, there was a 3-fold decrease in $P$. falciparum cases (from 2006 to 2010, 21,008 to 6,196 cases, respectively). Plasmodium vivax cases decreased nearly 2 -fold, but the decline was delayed (from 2006 to $2011,11,914$ to 6,642 cases, respectively) in comparison with $P$. falciparum (Figure 1). During this time, $P$. vivax transitioned to become the dominant species ${ }^{6}$ (Figure 2). After 2010, malaria positivity continued to decrease for both species. This occurred alongside a $~ 50 \%$ decline in consultations for non-malarial fever from 2011 to 2014 and which plateaued after 2014. In 2014, 2015, and 2016, approximately 3,400, 6,600 , and 6,200 cases, respectively, of $P$. vivax were treated by the community-based malaria clinics; many located a few days travel inside Myanmar. Migrants had a 50\% higher risk for malaria (incidence risk ratio (IRR) 1.5, 95\% Cl: 1.40-1.67; $P<0.001$ ) than refugees. In both migrant and refugee clinics, the greatest declines in malaria incidence risk occurred in the $<10$ year age-groups (Figure 3 ) and nearly an $80 \%$ reduction for $P$. falciparum (2006-2010) and $70 \%$ reduction for $P$. vivax (2006-2011) in the 0-4 years group. Females had a 56\% lower incidence risk of malaria per year (IRR 0.44, 95\% Cl: 0.40-0.49; $P<0.001$ ) (Supplemental Table 1). When assessing IRR differences between gender by age-group, males aged $\geq 10$ years had a higher incidence risk of $P$. falciparum and $P$. vivax malaria each year. This risk difference ended with the $\geq 40$ year age-group for $P$. vivax but persisted for $P$. falciparum (Supplemental Tables 2 and 3). The overall trends and risks
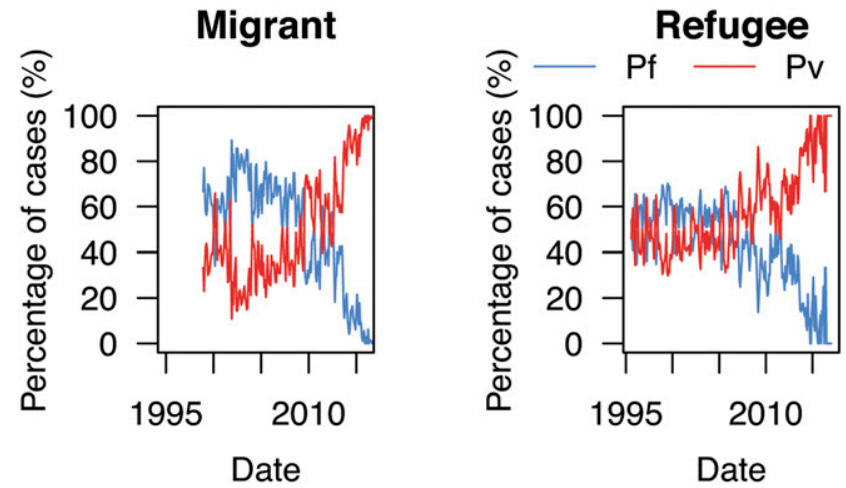

FIGURE 2. Percentage of Plasmodium falciparum and Plasmodium vivax for each year from 1998 to 2016 in migrant clinics and 1995 to 2016 in refugee clinics. 


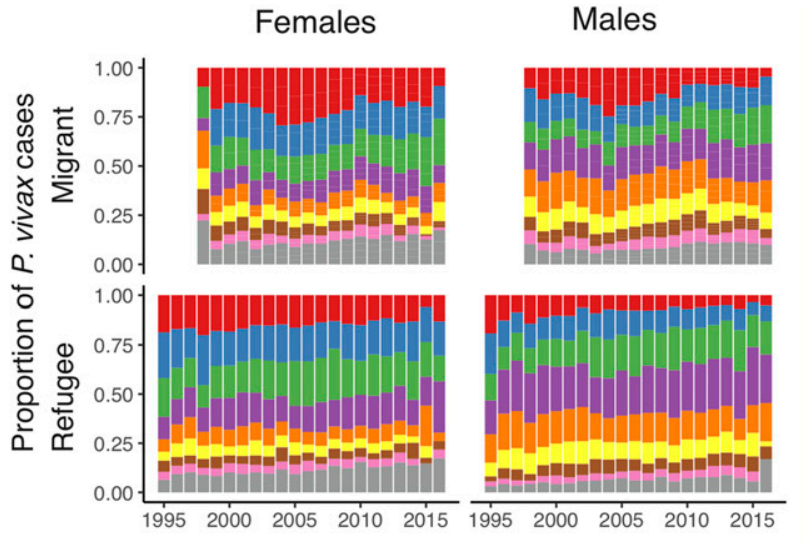

Year

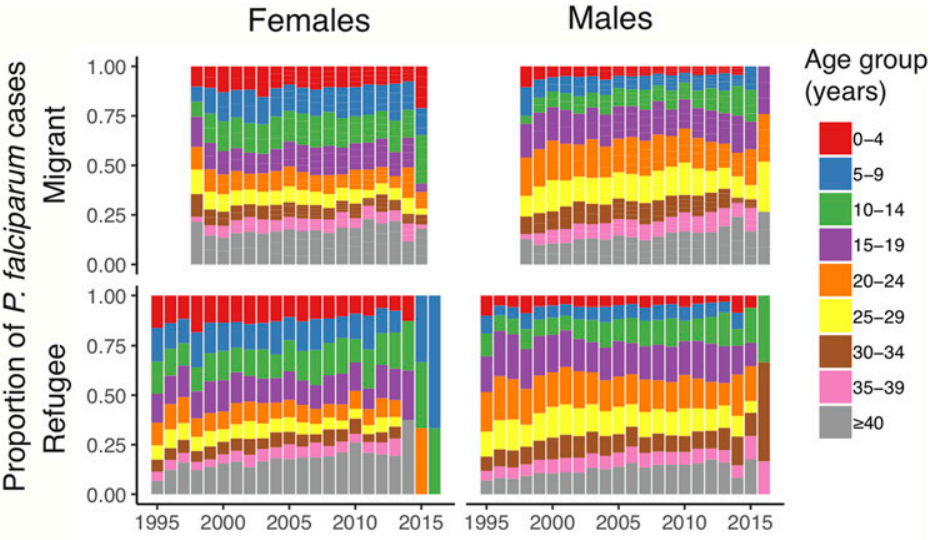

Year

FIGURE 3. Annual proportions of Plasmodium falciparum and Plasmodium vivax by age and year, stratified by gender from 1998 to $2016 \mathrm{in} \mathrm{migrant}$ clinics and 1995 to 2016 in refugee clinics. In 2016, there were three refugee and no migrant female cases of Plasmodium falciparum.

were similar when the analysis was performed with and without correcting for clinical $P$. vivax diagnosis.

\section{DISCUSSION}

As $P$. falciparum cases have decreased, a coincidental and delayed decrease in $P$. vivax has followed. ${ }^{9,10}$ Similar to other parts of Southeast Asia where $P$. falciparum is being eliminated, $P$. vivax becomes dominant, although not all countries report consistent declines in $P$. vivax caseload. ${ }^{11}$ Early diagnosis, efficacious ACTs, and single-dose primaquine contribute to lower $P$. falciparum transmission ${ }^{12,13}$; however, in the absence of widespread radical cure, the explanation for declining $P$. vivax is less clear.

The observed trend was not affected by the introduction of pan-malaria RDTs in 2010 . It is also unlikely that the provision of radical cure to $<10 \%$ of $P$. vivax patients would drastically reduce the incidence risk of $P$. vivax as demonstrated here. A more probable explanation to the decreasing $P$. vivax cases at SMRU medical clinics is the opening of more than 1,200 community-based malaria clinics along the border, where similar shifts have been reported ${ }^{14}$; however, radical cure for $P$. vivax is not given there. It is also possible that reducing $P$. falciparum transmission reduces $P$. vivax infections mainly by decreasing relapse activation. ${ }^{15,16}$

Other factors potentially contribute to the decline of $P$. vivax. From 2009 to 2016 , more than 280,000 insecticidetreated bed nets were distributed in the area, but their effectiveness is decreased by early evening mosquito biting behavior. ${ }^{17}$ Broad changes in the landscape along the international border, including deforestation, industrial agriculture, and urbanization, ${ }^{18,19}$ may contribute to an overall decrease in malaria, although a direct association cannot be made with these data. Gametocytocidal treatment with ACTs may contribute to decreased $P$. vivax transmission ${ }^{20}$; however, mixed infections are uncommon. A series of clinical studies (2010-2014) using radical curative primaquine may have contributed to decreased $P$. vivax transmission. This does not explain the longitudinal trends reported here or from the community clinics where no $P$. vivax studies are being conducted. ${ }^{8}$ Still, migrants and adult males remain at highest risk for $P$. vivax infection because of environmental and occupational exposure. Refugees (unless migratory) are confined to semi-urban camps where there is no malaria transmission. More detailed analyses stratified by age and gender are not possible with the available data.

In combination, species-specific diagnostics, bed nets, efficacious schizonticidal treatment, and comprehensive radical cure would presumably reduce the incidence of $P$. vivax at the same rate as $P$. falciparum. Without widespread radical cure, we postulate that the coincidental decline in $P$. vivax and its delay relative to $P$. falciparum in this area are due to decreased activation of $P$. vivax hypnozoites from reduced $P$. falciparum transmission. ${ }^{15,16}$ As the region approaches $P$. falciparum elimination, it is clear that $P$. vivax also requires a program for elimination. The trends reported here are promising, although radical cure will likely be necessary to completely eliminate $P$. vivax from this region.

Received July 2, 2019. Accepted for publication October 4, 2019.

Published online November 18, 2019.

Note: Supplemental tables appear at www.ajtmh.org.

Acknowledgments: This work was possible because of the trust the patients put into the hands of the competent and committed SMRU clinical teams. We also thank the SMRU computer team who have, for over 30 years, dedicated themselves to the management of data in a rapidly changing landscape of technology.

Financial support: Shoklo Malaria Research Unit is supported by the Wellcome Trust.

Authors' addresses: Cindy S. Chu, Rose McGready, and François Nosten, Shoklo Malaria Research Unit, Mahidol-Oxford Tropical Medicine Research Unit, Faculty of Tropical Medicine, Mahidol University, Mae Sot, Thailand, and Centre for Tropical Medicine, Nuffield Department of Medicine, University of Oxford, Oxford, United Kingdom, E-mails: cindy@tropmedres.ac, rose@shoklo-unit.com, and francois@tropmedres.ac. Verena I. Carrara, Department of Medicine, Swiss Tropical and Public Health Institute, Basel, Switzerland, E-mail: verena@shoklo-unit.com. Daniel M. Parker, Department of Population Health and Disease Prevention, University of California, Irvine, CA, and Department of Epidemiology, University of California, Irvine, CA, E-mail: dparker1@uci.edu. Stéphane Proux, Shoklo Malaria Research Unit, Mahidol-Oxford Tropical Medicine Research Unit, Faculty of Tropical Medicine, Mahidol University, Mae Sot, Thailand, E-mail: steph@shoklo-unit.com. Prakaykaew Charunwatthana, MahidolOxford Tropical Medicine Research Unit, Faculty of Tropical Medicine, Mahidol University, Mae Sot, Thailand, and Department of Clinical 
Tropical Medicine, Faculty of Tropical Medicine, Mahidol University, Bangkok, Thailand, E-mail: jib@tropmedres.ac.

This is an open-access article distributed under the terms of the Creative Commons Attribution License, which permits unrestricted use, distribution, and reproduction in any medium, provided the original author and source are credited.

\section{REFERENCES}

1. World Health Organization, 2016. Eliminating Malaria in the Greater Mekong Subregion: United to End a Deadly Disease. Geneva, Switzerland: WHO.

2. Chu CS et al., 2018. Comparison of the cumulative efficacy and safety of chloroquine, artesunate, and chloroquine-primaquine in Plasmodium vivax malaria. Clin Infect Dis 67: 1543-1549.

3. World Health Organization, 2015. Guidelines for the Treatment of Malaria, 3rd edition. Geneva, Switzerland: WHO.

4. Saita S, Silawan T, Parker DM, Sriwichai P, Phuanukoonnon S, Sudathip P, Maude RJ, White LJ, Pan-Ngum W, 2019. Spatial heterogeneity and temporal trends in malaria on the ThaiMyanmar border (2012-2017): a retrospective observational study. Trop Med Infect Dis 4: E62.

5. Luxemburger C, Thwai KL, White NJ, Webster HK, Kyle DE, Maelankirri L, Chongsuphajaisiddhi T, Nosten FH, 1996. The epidemiology of malaria in a Karen population on the western border of Thailand. Trans R Soc Trop Med Hyg 90: 105-111.

6. Carrara VI et al., 2013. Malaria burden and artemisinin resistance in the mobile and migrant population on the Thai-Myanmar border, 1999-2011: an observational study. PLoS Med 10: e1001398.

7. Parker DM, Landier J, Thu AM, Lwin KM, Delmas G, Nosten FH, 2017. Scale up of a Plasmodium falciparum elimination program and surveillance system in Kayin State, Myanmar. Wellcome Open Res 2: 98.

8. Landier J, Parker DM, Thu AM, Lwin KM, Delmas G, Nosten FH; for the Malaria Elimination Task Force Group, 2018. Effect of generalised access to early diagnosis and treatment and targeted mass drug administration on Plasmodium falciparum malaria in eastern Myanmar: an observational study of a regional elimination programme. Lancet 391: 1916-1926.

9. Maude RJ et al., 2014. Spatial and temporal epidemiology of clinical malaria in Cambodia 2004-2013. Malar J 13: 385.
10. Soto-Calle V et al., 2017. Spatio-temporal analysis of malaria incidence in the Peruvian Amazon region between 2002 and 2013. Sci Rep 7: 40350.

11. Chhetri V, Dukpa T, Namgay R, Wangchuk K, Pokrel HP, 2019. Burden of malaria in three endemic districts of Bhutan: road to malaria elimination. Int J Innov Res Med Sci 4: 65-70.

12. Carrara VI et al., 2006. Deployment of early diagnosis and mefloquine-artesunate treatment of falciparum malaria in Thailand: the Tak Malaria Initiative. PLoS Med 3: e183.

13. Landier J, Parker DM, Thu AM, Carrara VI, Lwin KM, Bonnington CA, PukrittayakameeS, Delmas G, Nosten FH, 2016. The role of early detection and treatment in malaria elimination. Malar J 15: 363.

14. METF. 2017. Malaria Elimination Task Force (METF) Activity Report: May 2014-December 2016. Mae Sot, Thailand.

15. Ashley EA, Phyo AP, Carrara VI, Tun KM, Nosten F, Smithuis F, White NJ, 2019. Plasmodium vivax relapse rates following Plasmodium falciparum malaria reflect previous transmission intensity. J Infect Dis 220: 100-104.

16. Nosten FH, van Vugt M, Price RN, Luxemburger C, Thway KL, Brockman A, McGready RM, ter Kuile FO, Looareesuwan S, White NJ, 2000. Effects of artesunate-mefloquine combination on incidence of Plasmodium falciparum malaria and mefloquine resistance in western Thailand: a prospective study. Lancet 356: 297-302.

17. Parker DM, Carrara VI, Pukrittayakamee S, McGready R, Nosten $\mathrm{FH}, 2015$. Malaria ecology along the Thailand-Myanmar border. Malar J 14: 388.

18. Johnson BA, 2015. Combining national forest type maps with annual global tree cover maps to better understand forest change over time: case study for Thailand. Appl Geogr 62: 294-300.

19. Li P, Feng Z, Xiao C, Boudmyxay K, Liu Y, 2018. Detecting and mapping annual newly-burned plots (NBP) of swiddening using historical Landsat data in Montane Mainland Southeast Asia (MMSEA) during 1988-2016. J Geogr Sci 28: 1307-1328.

20. Popovici J, VantauxA, Primault L, Samreth R, Piv EP, Bin S, Kim S, Lek D, Serre D, Menard D, 2018. Therapeutic and transmissionblocking efficacy of dihydroartemisinin/piperaquine and chloroquine against Plasmodium vivax malaria, Cambodia. Emerg Infect Dis 24: 1516-1519. 